\title{
Riesgo comparativo de reacción anafiláctica asociada a hierro endovenoso
}

Comparative risk of anaphilactic reactions associated with intravenous iron products

Wang C y col. JAMA 2015; 314 (19): 2062-2068.

\section{Objetivos}

Comparar el riesgo de anafilaxia de las diferentes presentaciones de hierro endovenoso.

\section{Diseño, lugar y pacientes}

Estudio de cohorte retrospectivo de usuarios del programa Medicare (EE.UU.) que entre 2003 y 2013 recibieron por primera vez hierro endovenoso (dextrano, gluconato, sacarosa o ferumoxitol) en forma ambulatoria ( $\mathrm{n}: 688.183$ ), sin estar bajo tratamiento dialítico.

\section{Medición de resultados principales}

Las reacciones anafilácticas fueron identificadas a través de un algoritmo pre-especificado y validado. El riesgo absoluto y relativo de desarrolar anafilaxis para cada una de las sales de hierro endovenoso fue estimado ajustando los disbalances entre los diferentes grupos de tratamiento.

\section{Resultados principales}

Se describen en la tabla 1.

Tabla 1. Riesgo de desarrollo anafilaxia durante la primera administración de hierro intravenoso.

\begin{tabular}{|c|c|c|c|c|c|c|c|c|c|}
\hline & \multicolumn{2}{|c|}{2003 a 2013} & \multicolumn{3}{|c|}{2003 a 2013} & \multicolumn{4}{|c|}{2010 a 2013} \\
\hline & Nodextran & Dextran & Sacarosa & Gluconato & Dextran & Sacarosa & Ferumoxitol & Gluconato & Dextran \\
\hline Casos de anafilaxia & 107 & 167 & 45 & 34 & 167 & 21 & 28 & 16 & 66 \\
\hline Nuevos pacientes & 440683 & 247500 & 264166 & 94400 & 247500 & 134836 & 82117 & 34029 & 77935 \\
\hline $\begin{array}{l}\text { Incidencia por cada } \\
100.000 \text { personas } \\
\text { (IC } 95 \%)\end{array}$ & $\begin{array}{c}24,3 \\
(20,0 \text { a } 29,5)\end{array}$ & $\begin{array}{c}67,5 \\
(57,8 \text { a } 78,7)\end{array}$ & 17 & 36 & $\begin{array}{c}67,5 \\
(57,8 \text { a } 78,7)\end{array}$ & 15,6 & $\begin{array}{c}34,1 \\
(23,1 \text { a } 50)\end{array}$ & $(27,8$ a 78,2$)$ & $\begin{array}{c}84,7 \\
(66 \text { a 108,4) }\end{array}$ \\
\hline $\begin{array}{l}\text { OR ajustado } \\
\text { (IC 95\%) } \\
\text { p }\end{array}$ & 1 & $\begin{array}{c}2,6 \\
(2,0 \text { a } 3,3) \\
<0,001\end{array}$ & 1 & $\begin{array}{c}2 \\
(1,2 \text { a } 3,5) \\
0,005\end{array}$ & $\begin{array}{c}3,6 \\
(2,4 \text { a } 5,4) \\
<0,001\end{array}$ & 1 & $\begin{array}{c}2,2 \\
(1,1 \mathrm{a} 4,3) \\
0,02\end{array}$ & $\begin{array}{c}3 \\
(1,4 \mathrm{a} 6,5) \\
0,001\end{array}$ & $\begin{array}{c}5,4 \\
(3,0 \text { a } 9,8) \\
<0,001\end{array}$ \\
\hline
\end{tabular}

\section{Conclusiones}

En pacientes que no están en diálisis, la incidencia de anafilaxia aso- ciada a recibir hierro endovenoso por primera vez es más alta con hierro dextrano y más baja con hierro sacarosa.

\section{Comentario}

Más de un cuarto de la población mundial padece anemia, la mitad debida a deficiencia de hierro.

Hay numerosos escenarios en los cuales el uso de hierro endovenoso es preferible al oral': pacientes con intolerancia digestiva, preferencia de lograr la repleción de sus depósitos de hierro en solo dos visitas, pérdidas de sangre activas que exceden la capacidad de repleción por la dieta, malabsorción y estados inflamatorios que interfieren con la homeostasis del hierro ${ }^{2}$, etc. Hay numerosas fórmulas de hierro endovenoso disponibles en el mercado, todas igualmente efectivas para el tratamiento de dicha deficiencia ${ }^{3}$. La frecuencia de los eventos adversos serios es comparable entre ellas y no debería ser un factor para la elección del producto, con la excepción del hierro dextrano de alto peso molecular, no disponible actualmente en el mercado ${ }^{4}$. En el estudio que hemos resumido, la frecuencia de reacciones serias podría estar sobreestimada debido a que los criterios de anafilaxia estaban basados en datos médicos codificados que no permitían al investigador determinar si los pacientes habían recibido antihistamínicos antes o después de la administración del hierro. Por otro lado, DeLoughery sostiene que la tasa de reacciones del hierro dextrano podría estar sobreestimada cuando se la pone en perspectiva con los estándares actuales de prescripción, ya que casi un $10 \%$ de los pacientes incluidos en esta cohorte retrospectiva habían recibido hierro dextrano de alto peso molecular, forma que ya casi no se utiliza ${ }^{5}$.

\section{Conclusiones de la comentadora}

El hierro endovenoso tiene el potencial de causar reacciones alérgicas, algunas con riesgo de vida. Sin embargo, estas son extremadamente raras y la frecuencia es similar para todos los productos usados en la actualidad, dado que ya no suele usarse el hierro dextrano de alto peso molecular.

Georgina Bendek Del Prete [ Departamento de Clínica Médica. Sección hematología. Hospital Italiano Agustín Roca de San Justo. georgina.bendek@ hospitalitaliano.org.ar]

Bendek Del Prete G. Riesgo comparativo de reacción anafiláctica asociada a hierro endovenoso. Evid Act Pract Ambul. 2017. 20(4):98. Comentado de: Wang C, y col. Comparative risk of anaphylactic reactions associated with intravenous iron products. JAMA. 2015;314(19):2062-2068. PMID: 26575062.

\section{Referencias}

1. Camaschella C. Iron Deficiency anemia, N England J Med 2015; 372: 1832.

2. Auerbach M y col. How we diagnose and Treat iron deficiency anemia, Am J Hematol. 2016; 91:31.

3. Röhring G y col. Efficacy and tolerability of ferric carboxymaltose in geriatric patients with anemia. Data from three non interventional studies. MMW Fortschr Med. $2014 ; 156$ Supl 2:48.

4. Camaschella C. Iron deficiency anemia. N Engl J Med. 2015;372:1832.

5. De Loughery T. Is low Molecular weight iron dextran really the most risk iron? Unconvincing data from an unconvincing study. Am J Haematol 2016; $91: 451$. 